\title{
Extraction of Blood Vessels in Ophthalmic Color Images of Human Retinas
}

\author{
Edgardo Felipe-Riveron ${ }^{1}$ and Noel Garcia-Guimeras ${ }^{2}$ \\ ${ }^{1}$ Centre for Computing Research, National Polytechnic Institute, \\ Juan de Dios Batiz s/n, P.O. 07038, Mexico \\ ${ }^{2}$ Latin American School of Medicine. Havana, Cuba \\ edgardo@cic.ipn.mx, noelgarciaguimeras@yahoo.com
}

\begin{abstract}
This paper presents a strategy for the extraction of blood vessels from ophthalmoscopic color images of the fundus of human retinas. To extract the vascular network, morphology operators were used, primarily maximum of openings and sum of valleys, and secondly a reconstruction by dilation from two images obtained using threshold by hysteresis. To extract the skeleton of the resulting vascular network, morphological thinning and pruning algorithms were used. Results obtained represent a starting point for future work related to the detection of anomalies in the vascular network and techniques for personal authentication.
\end{abstract}

Keywords: Blood vessels segmentation, Fundus analysis, Morphology.

\section{Introduction}

The automatic analysis of human eye fundus images is a task of major importance. The fundamental aim is to help ophthalmologists during the diagnosis of illnesses that present visible affections in the human retina. Some of these illnesses are of concern, because if they are not detected in time, they can lead to blindness. Of particular interest are: glaucoma, diabetic retinopathy, and macular degeneration. Early detection of these conditions is a focus of importance for ophthalmologists.

There are three visible anatomical elements in the fundus of the retina: the macula lutea, the optic disk (or optic papilla), and the vascular network, composed of a thick and dark red vein network, and a thinner arterial network of a clearer reddish tone (Fig. 1).

In order for the ophthalmologist to analyze the retina it is common to induce certain conditions on patients to improve the quality of observation of the eye fundus. To enlarge the observation field the pupils of the patients are dilated; to highlight affections of the retina related with blood, it is common to inject patients with a fluoresceinic substance that makes blood vessels visible with easiness. In this paper we propose a method to detect blood vessels in human retinas from digital ophthalmoscopic color images. 


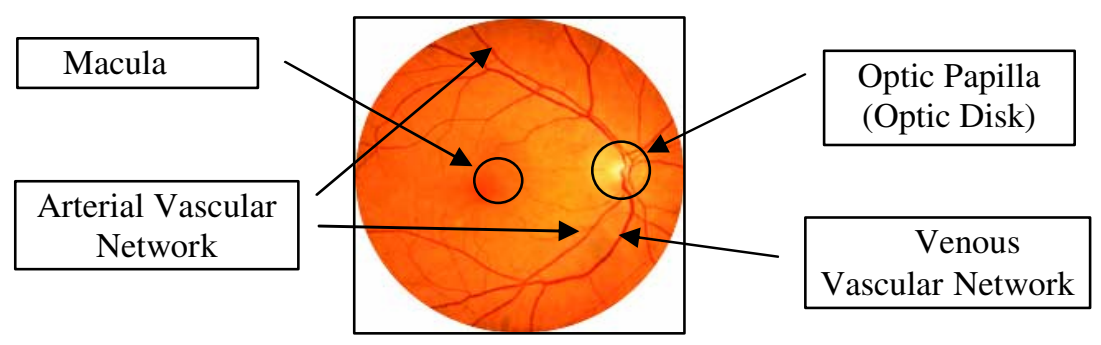

Fig. 1. Anatomic elements of a human eye fundus image

\section{The Problem}

Our task deals with the extraction of the vascular network from the retina. Due to the normal distribution through the entire retina and the probable non-uniform illumination of the field observed when retina images are captured, this is not a trivial task. Any strategy applied to achieve this purpose should be independent of nonuniform illumination and invariant to translation, rotation, and scale of the images being analyzed.

\section{Background}

The successful extraction of blood vessels from images of the retina has been treated previously. Filtering by coincidence has being used to separate the blood vessels in eye fundus images [1] [2]. As in their traverse section the blood vessels have the form of an overturned Gauss bell, their segmentation can be carried out by the convolution of images with a Gaussian nucleus. This method used by Chutatape and Zheng [3] is slow because it requires a large Gaussian mask for the convolution, and images need to be rotated several times. Also, the selected mask responds well when it represents the same standard deviation than that of pixels of blood vessels, but it does not respond either for smaller nor bigger blood vessels.

Artificial neural networks have been applied with promising results in the analysis of ophthalmic images [4] [5] [6]. They have demonstrated to be useful, since they can be trained to recognize patterns of blood vessels. In this way it is possible to extract them. A disadvantage of neural networks is that classification is carried out on the basis of statistical methods and logical reasoning.

Algorithms for tracking blood vessels have been used to segment them between two given points [7]. These algorithms work at the level of a single blood vessel and not of the whole vascular network. Usually, while moving through the blood vessel, these algorithms calculate their width, which represents an advantage. Other parameters like the width average and the tortuosity of the blood vessel can also be calculated. The main advantage of this method is that it gives quite exact measures and information about individual blood vessels. Unfortunately, they require a starting point and in most case a terminal point, which works against automation of the process. 
Image processing by means of morphological methods is particularly effective when it is required to extract components of the image whose shape is known a priori [8]. The vascular network is linear in small portions, which is ideal for morphological processing. Morphological methods have been applied in segmentation of certain types of vascular networks with the intrinsic advantage of being more efficient and more immune to noise than other methods. In particular, this approach has been applied to gray-level angiographic images captured after patients were injected with a fluoresceinic substance that highlights areas containing blood, as is the case with veins and arterial blood vessels of the vascular network [8] [9].

As mentioned earlier, this paper deals with the extraction of the vascular network from color ophthalmoscopic fundus images. These are images obtained without the necessity of invasive procedures.

\section{Our Strategy}

The method proposed for the extraction of the vascular network is based on methods proposed by Flynn [10], and by Zana and Klein [9]. The main difference with our strategy is that those methods work well for fluoresceinic gray-level images of retina where blood vessels appear with a higher intensity level and have well-defined contours. In this paper, images were captured from patients that did not require any previous invasive preparation, that is, our solution use color images captured directly and transformed to gray level images.

These images typically are corrupted by significant additive noise that causes the image background to have a non homogeneous gray level. We call background in this paper all parts of the image that do not offer useful information related to our task. On the other hand, the background's gray level is not uniform in all areas through which the vascular network appears. In the region of the optic disk and near to it, the background has a clearer tone of gray, while in regions near the macula the background appears darker. In this aspect, factors related to the illumination of images when they are captured, produce more illuminated areas and also areas where it is not possible to distinguish the location of blood vessels (Fig. 2).

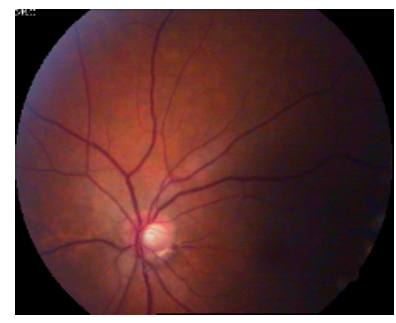

Fig. 2. Human eye fundus image

Generally, blood vessels are darker than the background, although there are areas where the vascular network is not visible because its level is similar to the background. The thickness of blood vessels is variable. In the area near the optic disk, 
where the two main veins and the two main arteries arise from the choroids through the center of the papilla to the retina, the vascular network is thicker. Other veins and arteries derived from these four are thinner, up to regions where almost they get lost when their thickness is only of one pixel. The last interesting characteristic of the vascular network is its piecewise linear structure. We can say that the vascular network is composed by small linear segments connected to each other [10]. The area near the center of the macula lutea is physiologically avascular.

Before extracting blood vessels images are preprocessed to normalize their size, to reduce additive noise and to enhance the contrast. All images used were obtained from the same source and have a spatial resolution of $300 \mathrm{dpi}$. Images were normalized to 350 pixels in the longer side.

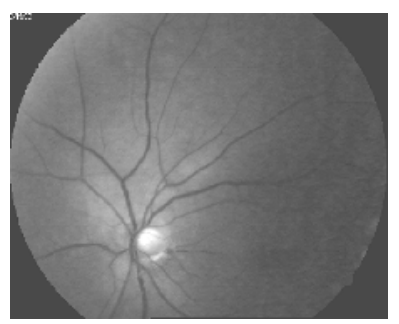

Fig. 3. Gray-level image from the green channel

Experimentally, as the green color is located at the centre of the visible spectrum, it has been observed that from the three-color channels that compose the image in the RGB (Red, Green, and Blue) color model, the green channel offers the higher quantity in information and less additive noise. The red channel still gives some information, and very little the blue channel, in occasions none, but having higher additive noise. Therefore, to avoid the influence of the noise present in these two channels, the conversion of the image to gray levels is based on the information contained in the green channel only (Fig. 3).

Subsequently, a morphological operator proposed by Zana and Klein [8], and Flynn [10] is applied, which diminishes at the same time the additive noise in the background and preserves blood vessels. The operator, called maximum of openings, is defined in the following way [12] [13]:

$$
I_{C}=\max _{\mathrm{i}=1 \ldots 16}\left\{I_{0}^{\circ}{ }^{\circ} B_{\mathrm{i}}\right\}
$$

Where $I_{\mathrm{C}}$ is the "cleaned" image, $I_{0}$ is the original image, and $B_{i}$ is a flat structuring element of height $h>0$ rotated 16 times. The size of the structuring element (SE) must be chosen in such a way that it preserves the blood vessels and eliminates the additive noise in the image. In our case, we have determined experimentally that 9 pixels length and 5 level unit's height SE gives the best results. 

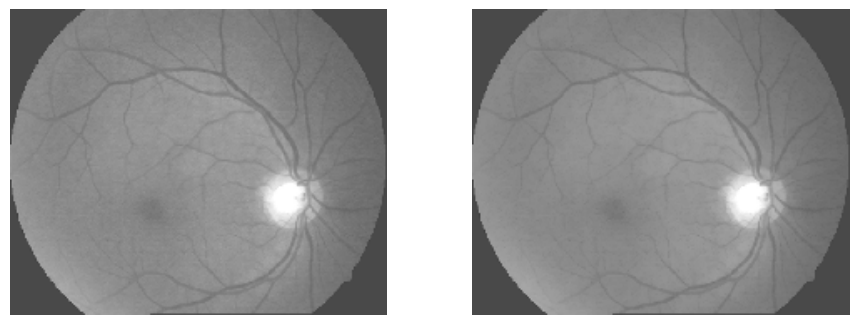

Fig. 4. Effects of the maximum of openings on the background of the image. (a) Original image (green plane). (b) Uniform background, while the structure of the blood vessels is preserved.

Note that after using this filter, the obtained image preserves blood vessels and have a more uniform background (Fig. 4b).

\subsection{Primary Detection of the Blood Vessels}

Once the image has been preprocessed, a preliminary detection of the blood vessels is carried out. The process is preliminary because in this step some artifacts remain, and have to be eliminated later. The operation is called sum of valleys defined as the dual of the operation proposed by Zana and Klein given previously [8]. The resulting image from this operation preserves blood vessels and is sharper, while the background spreads to be black. Figure 5(a) shows an example, in which the intensity has been inverted for clarity.

$$
I_{\text {sum }}=\sum_{i=1}^{16} \text { BOTHAT }\left(I_{C}, B_{i}\right)
$$
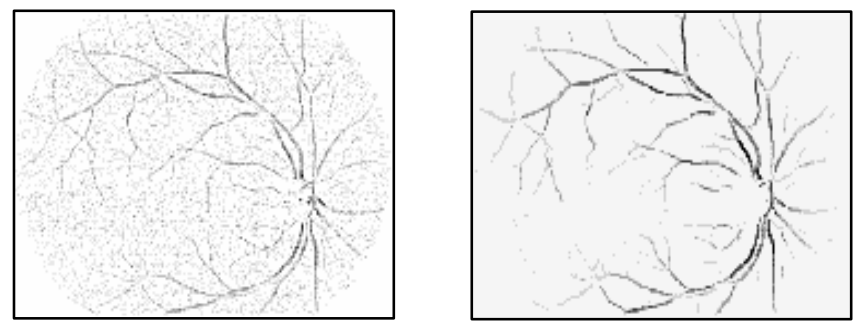

Fig. 5. (a) Image after the operator of sum of valleys is applied. Observe how noise and artifact are conserved in certain regions. (b) Image after suppression of noise and artifacts.

\subsection{Extraction of Blood Vessels}

The objectives of the next step are to suppress additive noise and artifacts, to preserve the vascular network and to prepare the image for converting it in a binary image.

Again, a maximum of openings is carried out to clean noise and artifacts, and preserve the useful information. The group of structuring elements is formed by a flat linear structure element rotated 16 times. As in previous operations, its longitude 
depends on the size of the blood vessels in the original image. In our case, two maximum of openings were carried out with structuring elements of different sizes and heights, in order to improve the extraction of the vascular network. Result of one of these maximum of openings is shown in Fig, $5 \mathrm{~b}$.

\subsection{Thresholding}

So far, part of the noise and artifacts still persist in the resulting images. They can be finally eliminated by means of thresholding by hysteresis and a reconstruction by dilation [13]. In this way, when the appropriate thresholds are chosen, the vascular network is isolated. We take advantage of the fact that the additive noise never reaches high gray levels, and that the vascular network is connected. The use of thresholding by hysteresis with an appropriate selection of minimum and maximum thresholds, allows eliminating the noise that still persists in the image, and obtaining a clean image with an isolated vascular network (Fig. 6).

(a)

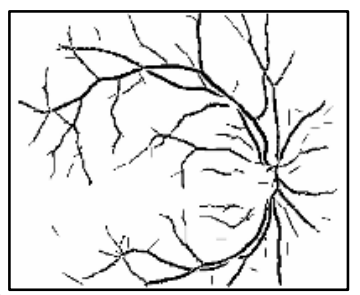

(b)

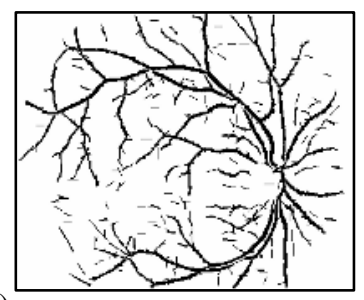

(c)

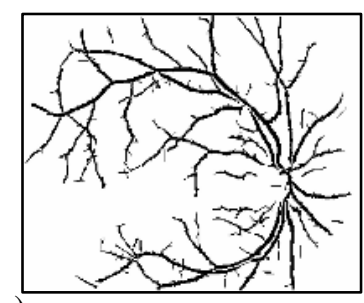

1. (b) Image with threshold in 6. (c) Image after reconstruction by dilation from previous images.

The image in Fig. 6(a) was obtained with threshold equal to 11. Notice the absence of noise, and that some blood vessels are not connected. This image is the binary mask. Fig. 6(b) shows the image with threshold equal to 6. Here the vascular network is fully connected, although there are also noise and artifacts. This is the binary marker. Figure 6(c) shows the image after being reconstructed by dilation using both the binary mask and the binary marker. Notice how the noise is reduced and the vascular network is now completely connected.

(a)

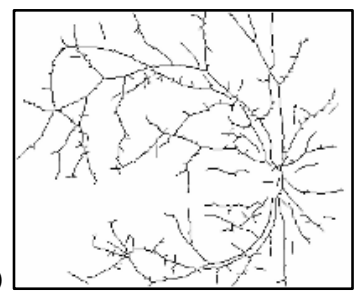

(b)

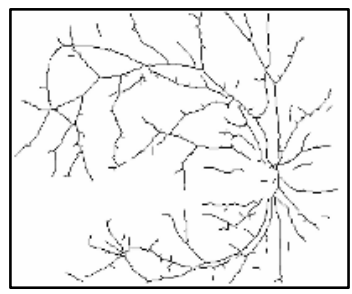

(c)

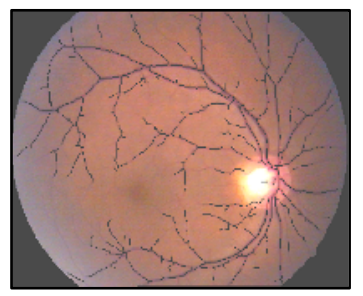

Fig. 7. (a) Skeleton of final image obtained from the process. (b) After pruning. (c) Segmented vascular network superimposed to the original image. 
The skeleton of the vascular network is obtained by means of the morphological operation of thinning. After it, the skeleton is not still smart, because it is common that it presents some non-desirable protuberances, which are eliminated later with several iterations of morphological pruning (Figs. 7a and 7b). In our case, two iterations were enough; finally, Fig. 7c shows the skeleton of the vascular network superimposed to the original image.

\section{Discussion of Results}

Some problems occurred in the detection of the vascular network due to its relative thickness and length with respect to the characteristics of the SE used. When the vascular network is thicker than the SE, it cannot be detected successfully. It is impossible to achieve a good segmentation if the size of the structuring elements is not adjusted. Generally it is common that all images proceed from the same source, by which once the SE is adjusted it does not present serious problems. In our case, the algorithm was applied to 11 color ophthalmic images with 24 bits/pixel originally, 9 from a Visucam-Lite, Kart Zeiss camera, and 2 from Internet network.

It can happen that once the sizes of structuring elements have been adjusted, there are regions where two blood vessels may be nearly parallel. In this case, they appear like a double cross sized blood vessel, and then a successful segmentation usually is not possible (Figs. 8a and b).

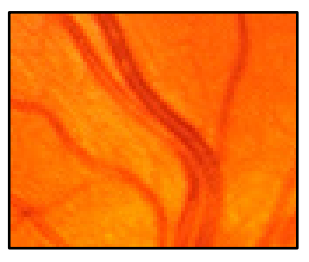

(a)

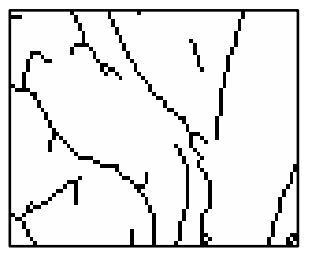

(b)

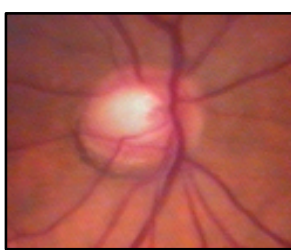

(c)

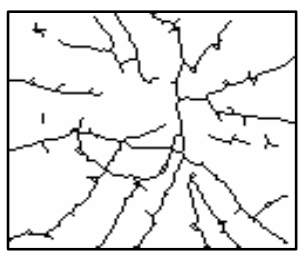

(d)

Fig. 8. (a) Fragment where two blood vessels appear very near. (b) Faulty detection. (c) Image where an area with atrophy is observed in the left inferior border of the optic disk. (d) False detection in this area.

Another problem is the false detection of the border of the optic disk. In a healthy eye this border is presented with a normal transition from clearer to darker shades of gray, and there is no problem. But some atrophy can be present due to glaucoma in the border of the optic disk. In these cases, false detections of the border of the optic disk can occur, as shown in Figs. 8c and 8d. This problem is unavoidable, due to the similar morphology of the blood vessels and the border of the optic disk.

We note that our results presented thus far were obtained without contrast enhancement. That was one of our objectives in order to process images as they were captured.

Osareh, Mirmehdi et al. have obtained good results with preprocessing steps related mainly to color normalization and contrast enhancement of retinal color images, using Fuzzy C-means clustering in the automatic recognition of exudative maculopathies [14]. 

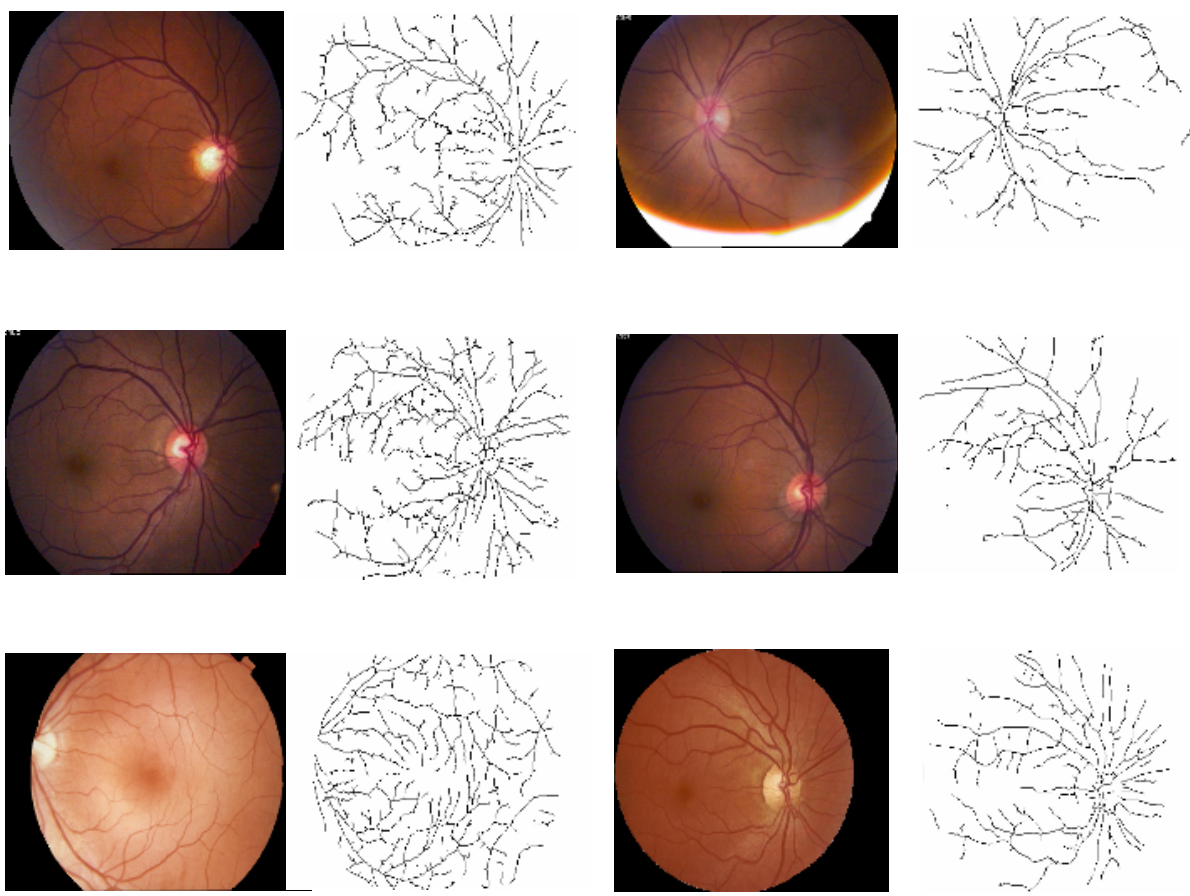

Fig. 9. Original images (left). The skeleton of the vascular network (right). Figures from (a) to (d) are real images. Figures (e) and (f) show images from Internet.

\section{Conclusions}

In this paper we implemented an algorithm for extracting the blood vessels from real color ophthalmoscopic images of the fundus of the human retina, using morphological methods. Images processed were captured in color without any previous bloody preparation of the patients with fluoresceinic substances to highlight the vascular network. The solution was based on two types of operators: maximum of openings and sum of valleys. The algorithm shows a favorable answer in the detection of blood vessels, although the presence of noise can affect the detection in some degree. The extraction is finally achieved using a reconstruction by dilation of two binary images obtained with two different thresholds. The skeleton was finally obtained by thinning and pruning the extracted vascular network.

\section{Acknowledgements}

The authors would like to thank the National Polytechnic Institute (Academic Secretary, COFAA, SIP, and the Centre for Computing Research), for their economical support to develop this work. 


\section{References}

1. Chaudhury, S., Chatterjee, S., Katz, N., Nelson, M., Goldbaum, M.: Detection of blood vessels in retinal images using two-dimensional matched filters, IEEE Transactions on medical imaging, Vol. 8, No. 3 (1989)

2. Hoover, A., Kouznetsova, V., Goldbaum, M.: Locating blood vessels in retinal images by piecewise threshold probing of matched filter response. IEEE Transactions on Medical Imaging, Vol. 19, No. 3 (2000)

3. Chutatape, O., Zheng, L., Krishnan, S. M.: Retinal blood vessel detection and tracking by matched gaussian and Kalman filters. Proceedings in the 20th Annual International Conference of the IEEE, Engineering in Medicine and Biology Society, Vol. 20, No. 6 (1998)

4. Gardner, G. G., Keating, D., Williamson, T.H., Elliot, A. T.: Automatic detection of diabetic retinopathy using an artificial neural network: a screening tool. British Journal of Ophthalmology (1996)

5. Undrill, P.: Towards the automatic interpretation of retinal images, British Journal of Ophthalmology, 80, 973 (1996)

6. Zana, F., Klein, J. C.: A multimodal algorithm of eye fundus images using vessel detection and Hough transform, IEEE Transactions on Medical Imaging. Vol. 18, No. 5 (1999)

7. Zhoue, I., Rzeszotarski, M., Singerman, L., Cokreff, J.: The detection and quantification of retinopathy using digital angiograms, IEEE Transactions on Medical Imaging, Vol. 13, No. 4 (1994)

8. Zana, F., Klein, J. C.: Robust segmentation of vessels from retinal angiography, Proceedings of the International Conference on Digital Signal Processing, Santorini, Greece (1997)

9. Zana, F., Klein, J. C.: Segmentation of vessel-like patterns using mathematical morphology and curvature evaluation. IEEE Transactions on Image Processing, Vol. 10, No. 7 (2001)

10. Flynn, J.: Automated vessel extraction in digital ophthalmic images.

11. Gang, L., Chutatape, O., Krishnan, S. M.: Detection and measurement of retinal vessels in fundus images using amplitude modified second-order gaussian filter. IEEE Transactions on Biomedical Engineering, Vol. 49, No. 2 (2002)

12. Gonzalez, R., Woods, R. E.: Digital Image Processing, Addison-Wesley, Imington, Delaware, USA (1996)

13. Soille P.: Morphological Image Analysis. Principles and Applications, Springer Verlag, Berlin (1999)

14. Osareh, A., Mirmehdi, M., Thomas, B., Markham, R.: Automatic Recognition of Exudative Maculopathy using Fuzzy C-Means Clustering and Neural Networks. Medical Image Understanding and Analysis (2001) 49-52 